\title{
Étude des paramètres de la dégradation dans le rumen des glucides pariétaux des aliments concentrés
}

\author{
P Chapoutot, D Sauvant, M Dorléans \\ INRA, station Nutrition et Alimentation, INA-PG, 16, rue Claude-Bernard, \\ 75231 Paris Cedex 05, France
}

Summary - Study of the degradation parameters of concentrate cell walls in the rumen. Adjustments of the kinetics of concentrate NDF degradation, measured at 13 incubation points, showed that the model proposed by Van Milgen was insufficient to accurately explain their variations. More sophisticated models therefore seem to be needed.

Le temps de latence qui intervient avant la fermentation ruminale des glucides, pariétaux en particulier, n'a été jusqu'à présent modélisé que de façon empirique (Mertens, 1973; Sauvant et al, 1986). Une formalisation plus mécaniste, qui intègre un compartiment de maturation disparaissant selon un taux $k_{1}$ au profit d'un compartiment de dégradation présentant lui-même un taux de dégradation $k_{d}$, a été proposée par Van Milgen et al (1991) et testée dans ce travail.

Matériel et méthodes - Dix aliments concentrés ont fait l'objet de mesure de dégradation dans le rumen, selon la méthode in situ présentée par Michalet-Doreau et al (1987) (3 vaches taries $\times 2$ répétitions; $70 \%$ foin pré $+30 \%$ concentré mixte). Sur les résidus d'incubation après $0,5,1,2,3,4,6,8,12,16,24,36$, 48 et $72 \mathrm{~h}$, le dosage du NDF a permis de mesurer les quantités résiduelles $\left(R_{t}\right)$ de la fraction pariétale. Celles-ci ont été ajustées par le modèle monocompartimental à temps de latence discret $(L): R_{1}=F_{d} e^{-k_{d}(t-L)}+F_{i}$, et par le modèle bicompartimental de Van Milgen et al (1991) :

$$
R_{t}=F_{d}\left|\frac{\left(k_{d} e^{-k_{i} t}-k_{l} e^{-k_{d} t}\right)}{\left(k_{d}-k_{l}\right)}\right|+F_{i},
$$

en fonction des fractions dégradable $\left(F_{d}\right)$ ou non $\left(F_{i}\right)$ et des taux de latence $\left(k_{1}\right)$ et de dégradation (kd).

Résultats et discussion - Les cinétiques de dégradation des parois cellulaires font apparaître, par le modèle classique monocompartimental, des temps de latence (L) importants pour CGM (28 h), TPA et TST (16-18 h), TSO et POI (11-13 h), PBE (7 h), comparées à ceux de LUD ( $2 \mathrm{~h}), \mathrm{MAl}$ et ORG (1 h), DRE (0 h). Pour les 10 aliments, le modèle bicompartimental aboutit à des valeurs $k_{d}$ et $k_{1}$ impossibles à distinguer statistiquement. La simplification de ce modèle par développement limité : $R_{t}=F_{d}$ $(1+k t) e^{-k t}+F_{i}$, où $k$ représente la valeur commune de $k_{d}$ et $k_{1}$, a conduit aux valeurs des paramètres présentées au tableau $I$. Les résidus réduits des ajustements des cinétiques des aliments ont été soumis à une analyse en composantes principales. Les 2 premières composantes, qui expliquent $63 \%$ de la variabilité, permettent d'établir une typologie en 3 groupes de matières premières. La figure 1 rapporte pour chacun les écarts réduits moyens, dont certains sont significativement différents de 0 compte tenu du nombre d'aliments par groupe. Le groupe A comprend les aliments à NDF dégradable (LUD, POI, PBE, TPA, TSO) qui présentent un temps de latence prolongé jusqu'à $12 \mathrm{~h}$, mal ajusté par le modèle, suivi par une dégradation plus rapide que celle montrée par le modèle. Le groupe $B$ correspond aux parois cellulaires du maïs (MAl et CGM), et au tourteau de soja tanné (TST) qui est proche de la frontière entre les groupes A et B. Ces aliments présen- 
tent un temps de latence encore plus prolongé (de 12 à $24 \mathrm{~h}$ ). Le groupe $\mathrm{C}$ inclut les parois de l'orge (ORG et DRE), qui se caractérisent par l'existence d'une fraction pariétale plus résistante que ne le suppose le modèle entre 6 et $36 \mathrm{~h}$ d'incubation.

En conclusion, le modèle à 2 compartiments associé à des processus du premier ordre proposé par Van Milgen apparaît insuffisant pour traduire l'hétérogénéité des processus de colonisation et de dégradation des constituants pariétaux des aliments. II conviendrait de tester des modèles à plus de 2 compartiments et/ou intégrant des processus non linéaires, le principal problème étant alors celui du nombre de points expérimentaux pour en déterminer avec précision les paramètres.

Mertens DR (1973) Ph D Thesis, Cornell Univ Ithaca, NY, $217 \mathrm{p}$

Michalet-Doreau $B$, Vérité $R$, Chapoutot $P$ (1987) Bull Tech CRVZ Theix, (69) 5-7

Sauvant $D$, Dorléans $M$, Delacour $C$, Bertrand D, Giger S (1986) Reprod Nutr Dév 26 (1B), 303-304

Van Milgen J, Murphy MR, Berger LL (1991) J Dairy Sci 74, 2515-2529

Tableau I. Paramètres de la dégradation in situ des parois cellulaires des aliments.

\begin{tabular}{|c|c|c|c|c|c|c|}
\hline Aliments & & $F i(\% M S)$ & $F d(\% M S)$ & $k\left(\% h^{-1}\right)$ & ETR (\% MS) & Groupes ${ }^{1}$ \\
\hline Luzerne déshydratée & (LUD) & 20,0 & 25,9 & 11,9 & 0,9 & A \\
\hline Drêches Brasserie & (DRE) & 24,0 & 47,7 & 11,1 & 2,5 & $\mathrm{C}$ \\
\hline Corn Gluten Meal & (CGM) & $-2,0$ & 17,7 & 5,7 & 1,7 & B \\
\hline Maïs grain & (MAI) & 1,6 & 9,9 & 8,6 & 0,6 & $B$ \\
\hline Orge & (ORG) & 7,9 & 11,9 & 16,0 & 1,2 & $\mathrm{C}$ \\
\hline Pois & (POI) & $-0,2$ & 11,7 & 10,5 & 0,9 & A \\
\hline Pulpe Betterave & (PBE) & 0,9 & 40,7 & 12,9 & 2,2 & A \\
\hline Tourteau Palmiste & (TPA) & 12,9 & 51,2 & 5,6 & 4,9 & $A$ \\
\hline Tourteau Soja & (TSO) & $-1,0$ & 21,7 & 9,8 & 3,1 & $A$ \\
\hline Tourteau Soja Tanné & (TST) & $-2,0$ & 33,0 & 7,3 & 3,2 & B \\
\hline
\end{tabular}

${ }^{1}$ Groupes établis à partir des écarts au modèle (voir texte et figure 1).

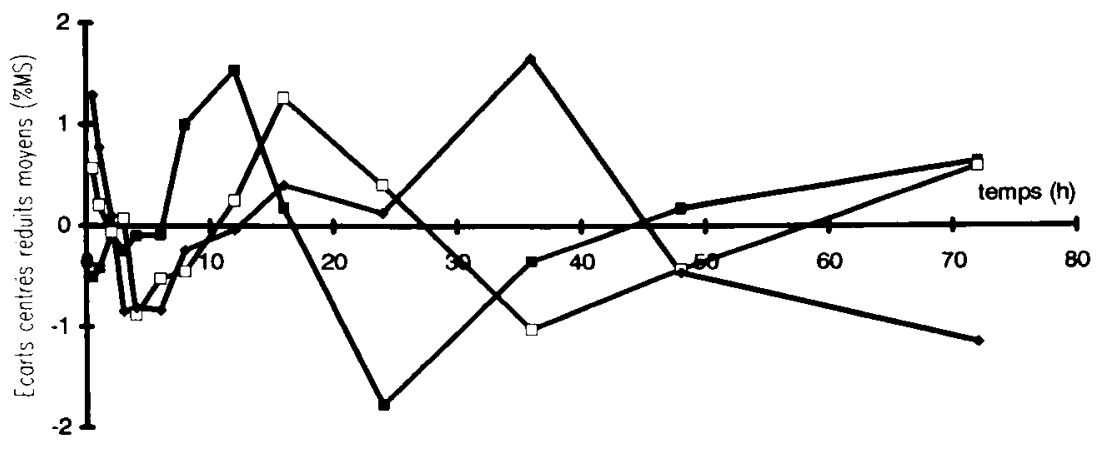

Fig 1. Évolution des écarts au modèle suivant les groupes d'aliments. $\mathrm{B} ;-\longrightarrow$ groupe $\mathrm{C}$. groupe A; $\square-$ - groupe 